\title{
Spectroscopy and Photodissociation of Dimethylzinc in Solid Argon. 1. Vacuum UV Luminescence Detection/Synchrotron Radiation Photolysis
}

\author{
Veronica A. Bracken, ${ }^{\dagger}$ Peter Guirtler, ${ }^{\ddagger}$ and John G. McCaffrey*,† \\ Department of Chemistry, National University of Ireland, Maynooth, Co. Kildare, Ireland, \\ and HASYLAB at DESY, Notkestrasse 85, 22603 Hamburg, Germany
}

Received: July 14, 1997; In Final Form: October 15, $1997^{\otimes}$

\begin{abstract}
Absorption spectra of thin-film DMZ/Ar samples, prepared by condensing gaseous mixtures of dimethylzinc (DMZ) with argon at $12 \mathrm{~K}$, were recorded in the region of the first dissociative absorptions of DMZ centered in the gas phase at $200 \mathrm{~nm}$. Large blue shifts are observed in the matrix spectra which can be related to the Rydberg-like characteristics of these excited states of DMZ. The photochemistry of DMZ in an argon matrix was investigated either by subjecting samples to undispersed synchrotron irradiation using a quartz filter to select a wavelength range above $155 \mathrm{~nm}$ or to wavelength-specific irradiation. Steady-state and time-resolved luminescence spectroscopy of the dissociation products isolated in solid argon indicate the existence of atomic zinc strongly perturbed by a methyl radical in freshly photolyzed samples, which yields truly isolated atomic zinc upon annealing to $33 \mathrm{~K}$. Dissociation threshold measurements indicate a barrier of $25 \mathrm{kcal} / \mathrm{mol} \mathrm{for}$ direct cage escape of atomic zinc in the Ar lattice. The increased intensity of $\mathrm{Zn}\left({ }^{3} \mathrm{P}_{1}\right) / \mathrm{Ar}$ emission observed in photolyzed DMZ/Ar samples relative to pure $\mathrm{Zn} / \mathrm{Ar}$ samples is explained in terms of the enhanced ISC of atomic zinc in the presence of hydrocarbon species in the former samples. This has been shown by codeposition of atomic zinc with Ar doped with $\mathrm{CH}_{4}$ and $\mathrm{C}_{2} \mathrm{H}_{6}$.
\end{abstract}

\section{Introduction}

Dimethylzinc belongs to a class of organometallic molecules known collectively as the dialkylmetals $\left(\mathrm{R}_{2} \mathrm{M}\right)$, where $\mathrm{R}=\mathrm{CH}_{3}$, $\mathrm{C}_{2} \mathrm{H}_{5}, \ldots$, and $\mathrm{M}=\mathrm{Zn}$, Cd, or Hg, whose characteristic of greatest technological significance is their photodissociation in the gas phase, producing metal atoms. The "direct writing technique", for example, a routine method utilized in the electronics industry for writing microcircuits on semiconductor materials, ${ }^{1}$ relies on the production of atomic zinc using a focused UV laser to dissociate dimethylzinc. As a result of its technological importance, the UV spectroscopy ${ }^{2}$ and photodissociation of DMZ in the gas phase ${ }^{3-5}$ and in molecular beams ${ }^{6}$ have been investigated by several groups, but to date, no work has been done in the solid state. In this and the following paper in this issue, the spectroscopy and photodissociation of DMZ isolated in solid Ar is examined with vacuum UV luminescence and FTIR absorption spectroscopies, respectively.

The photodissociation mechanism of DMZ in the gas phase has been examined in detail, ${ }^{4-6}$ leading to the proposal of the following stepwise mechanism for the production of atomic zinc:

$$
\begin{gathered}
\left(\mathrm{CH}_{3}\right)_{2} \mathrm{Zn} \rightarrow \mathrm{CH}_{3} \mathrm{Zn}+\mathrm{CH}_{3}^{\bullet} \\
\mathrm{CH}_{3} \mathrm{Zn}
\end{gathered}
$$

Vibrationally excited methylzinc and methyl radicals are produced after one UV photon absorption by DMZ, leading to the bent, first excited state and the linear, second excited electronic state. ${ }^{5}$ The nascent methylzinc radical immediately dissociates in the gas phase due to excess vibrational energy.

\footnotetext{
* To whom correspondence should be addressed.

${ }^{\dagger}$ National University of Ireland.

\# HASYLAB at DESY.

${ }^{\otimes}$ Abstract published in Advance ACS Abstracts, December 1, 1997.
}

The gas-phase work of Strausz and co-workers ${ }^{3}$ and more recently Jackson, ${ }^{4}$ in which methylzinc radicals were produced by the photolysis of dimethylzinc in the presence of a buffer gas, demonstrated that the yield of methlyzinc radical increased with increasing buffer gas pressure at the expense of zinc atom production. Miller and co-workers ${ }^{7}$ have recently exploited this behavior to produce methylzinc radical in a supersonic jet by laser photolysis of dimethylzinc under the high-collision conditions present in the throat of the expansion. Cooling and, hence, stabilization of methylzinc radical were facilitated by further expansion in the jet, allowing high-resolution spectroscopic analysis $^{7}$ of the ultracold methylzinc radical.

The vibrational relaxation trends exhibited in the gas phase work would indicate that the methylzinc radical should be efficiently stabilized in the solid. On this basis, it might then be expected that the photodissociation of DMZ in the solid would lead predominantly to the formation of methylzinc and a methyl radical. On the other hand, could geminate recombination arising from "cage effects" minimize the permanent production of such reactive radicals in the solid? Using IR spectroscopy in conjunction with steady-state and time-resolved luminescence spectroscopy as sensitive probes of dissociation, identification of the fragments in the cryogenic matrix will be used to answer the questions raised above. In addition, details of the vacuum UV and IR spectroscopy of DMZ and its photodissociation products in solid argon are presented in this and the following papers in this issue, ${ }^{8}$ respectively.

The format of this paper is as follows. In the first part, the vacuum UV absorption spectroscopy of matrix-isolated DMZ in $\mathrm{Ar}$ is presented. The photochemical fragments produced with synchrotron radiation photolysis are examined with UV luminescence spectroscopy. Assignments of the atomic and molecular photodissociation products of DMZ are then made from annealing experiments in conjunction with the FTIR study presented in the succeeding paper. ${ }^{8}$ From comparisons with 
the behavior observed in pure $\mathrm{Zn} / \mathrm{Ar}$ samples and $\mathrm{Zn} / \mathrm{hydrocarbon} /$ Ar samples, assignments of the fragments have been established. On the basis of the spectral assignments of the photochemical products, the dissociation mechanism of DMZ in solid $\mathrm{Ar}$ is discussed and compared to the gas phase in the final section.

\section{Experimental Section}

Steady-state and time-resolved optical measurements were conducted, as described previously, ${ }^{9}$ at the HIGITI experimental station in HASYLAB at DESY, Hamburg, using synchrotron radiation (SR) optimized in the vacuum UV spectral region from the W3 Mini-Wiggler of the DORIS III positron storage ring. Absorption spectra were recorded in the region of the $\mathrm{B} \leftarrow \mathrm{X}$ and $\mathrm{A} \leftarrow \mathrm{X}$ dissociative absorptions of DMZ, centered at 200 $\mathrm{nm}$ in the gas phase, ${ }^{5}$ by scanning with a $1 \mathrm{~m}$ normal incidencemodified Wadsworth monochromator and using a sodium salicylate converter and an XP2020 photomultiplier tube for photon detection.

Steady-state (emission and excitation) luminescence measurements were made with a secondary monochromator, using either a $0.4 \mathrm{~m}$ Seya-Namioka monochromator for measurements in the vacuum UV and near-UV regions or a $0.2 \mathrm{~m}$ Acton Research Corporation Type VM 502 monochromator for the UV-visible region. A Hamamatsu MCP 1645U-09 microchannel plate and a Hamamatsu R 943-02 photomultiplier tube were used as photon detectors on the Seya-Namioka and ARC monochromators, respectively. The detection system was operated in the single-photon-counting mode, allowing the measurement of emission decay curves, as described elsewhere, ${ }^{9}$ with time correlation of the pulsed synchrotron radiation, viz., timecorrelated single photon counting (TCSPC). Sample annealing was achieved with a heater mounted on the cold tip of the liquid He cryostat, and temperatures were measured using a Lakeshore Cryotronics silicon diode. During sample annealing, a Balzers QMG 112A quadrupole mass spectrometer was used to detect and identify the gases evolving from the photolyzed DMZ/Ar samples.

The photochemistry of DMZ in the cryogenic matrix was investigated by subjecting the samples to either wavelengthselected irradiation or to irradiation with undispersed synchrotron light (achieved by setting the primary monochromator to zero order) using a quartz optical filter to select wavelengths longer than $155 \mathrm{~nm}$.

\section{Results}

A. Electronic Absorption Spectroscopy. An absorption spectrum recorded in the ultraviolet spectral region at $8 \mathrm{~K}$ for a freshly deposited DMZ/Ar sample having a dilution ratio of $1 / 1000$ is shown in the upper panel of Figure 1. A broad featureless band centered at $183 \mathrm{~nm}$ and a weaker shoulder extending up to $220 \mathrm{~nm}$ are observed. These profiles resemble the absorption profile observed in the gas phase, ${ }^{5}$ shown in the lower panel of Figure 1, but are considerably blue-shifted in the gas-phase $\mathrm{B} \leftarrow \mathrm{X}$ absorption centered at $204 \mathrm{~nm}$ and the $\mathrm{A}$ $\leftarrow \mathrm{X}$ electronic transitions extending up to $240 \mathrm{~nm}$. Knowing the DMZ/Ar dilution ratios and the sample thickness, the absorption measurements allow extraction of the oscillator strengths of DMZ transitions in the solid Ar host. $f$ values of 0.262 and 0.591 were determined for the $\mathrm{B} \leftarrow \mathrm{X}$ and $\mathrm{A} \leftarrow \mathrm{X}$ transitions, respectively, from the Gaussian fits shown in Figure 1 , which compare well with the values of 0.302 and 0.475 observed in the gas phase. ${ }^{5}$ In contrast to the pure vibrational ${ }^{8}$ transitions of DMZ, presented in the following paper in this issue, the electronic transitions exhibit, as demonstrated in

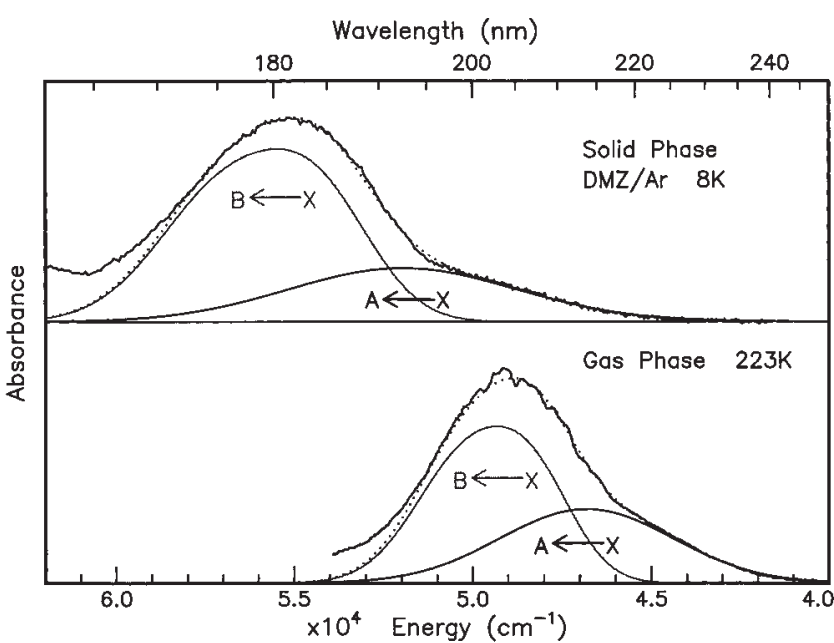

Figure 1. Absorption spectrum recorded at $8 \mathrm{~K}$ in the ultraviolet spectral region for a freshly deposited DMZ/Ar sample having a dilution ratio of $1 / 1000$ (upper panel). Shown in the lower panel are the $\mathrm{B}$ $\mathrm{X}$ and $\mathrm{A} \leftarrow \mathrm{X}$ electronic absorption transitions centered at $204 \mathrm{~nm}$ and extending up to $240 \mathrm{~nm}$, respectively, as recorded at $223 \mathrm{~K}$ in the gas phase by Chen and Osgood. ${ }^{5}$

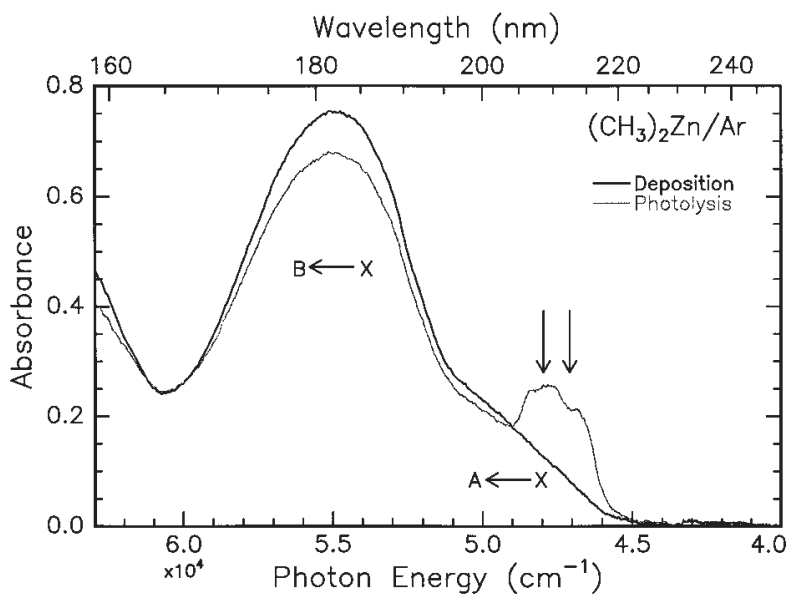

Figure 2. Absorption spectra, presented on a linear energy scale, of a matrix-isolated DMZ/Ar sample recorded at $8 \mathrm{~K}$ and prepared using a dilution ratio of $1 / 1000$. The black trace was recorded upon deposition, while the gray trace was recorded after the DMZ/Ar sample was irradiated for 15 min with undispersed synchrotron radiation using an quartz optical filter.

Figure 1, blue shifts on the order of several thousand wavenumbers in the Ar matrix, if the gas-phase assignments are adopted.

The vibrational structure present on the gas-phase $\mathrm{B} \leftarrow \mathrm{X}$ absorption is clearly absent in the solid state even at the very low temperature $(8 \mathrm{~K})$ at which the spectra in Figure 1 were recorded. Luminescence of DMZ in the UV-visible spectral range, covered in the present study, was not detected as a result of photoexcitation with synchrotron radiation at the $\mathrm{B} \leftarrow \mathrm{X}$ or $\mathrm{A} \leftarrow \mathrm{X}$ transitions of DMZ/Ar shown in the upper panel of Figure 1.

B. Zeroth Order Photolysis. Photochemistry was examined initially by subjecting DMZ/Ar samples to undispersed synchrotron radiation using a quartz optical filter to select wavelengths longer than $155 \mathrm{~nm}$. The effect of $15 \mathrm{~min}$ of this photolysis on the absorption profile is indicated by the gray trace shown in Figure 2. New features are observed between 205 and $220 \mathrm{~nm}$ in the red wing of the DMZ A $\leftarrow \mathrm{X}$ absorption upon photolysis in conjunction with a decrease in the intensity of both DMZ bands. 


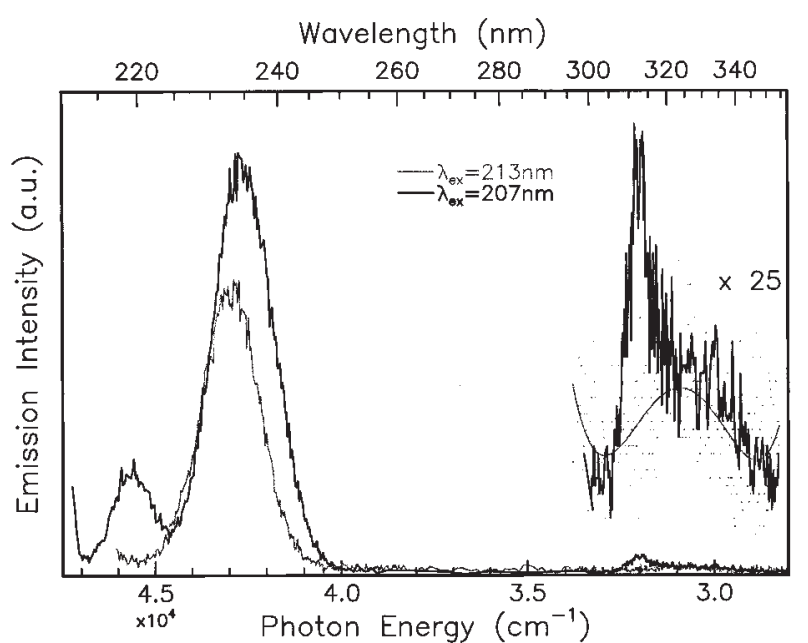

Figure 3. Luminescence spectra recorded at $8 \mathrm{~K}$ as a result of photoexcitation at 207 and $213 \mathrm{~nm}$ (indicated by the arrows in Figure 2) of the absorption features produced with photolysis of DMZ/Ar. The weak near-UV emission bands in the 300-340 nm spectral region are shown on an expanded vertical scale for ease of identification.
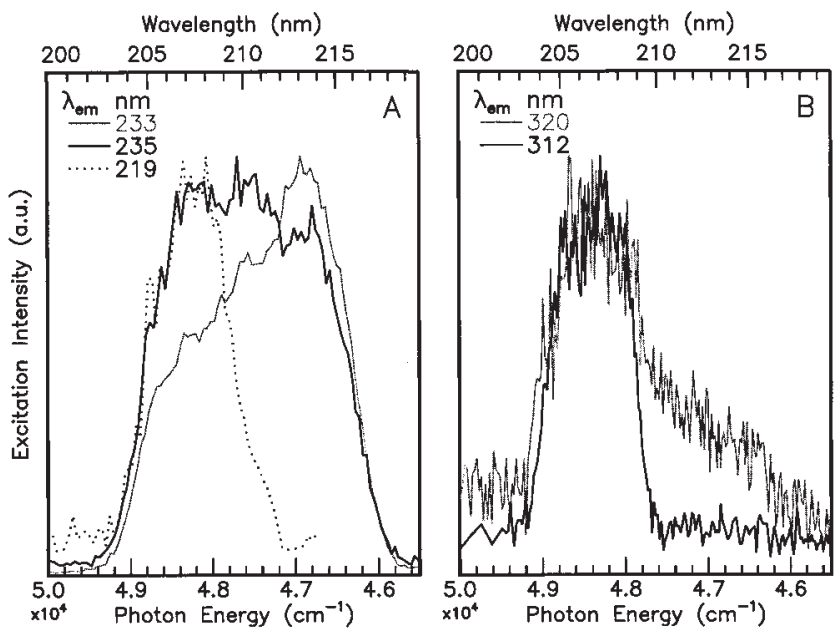

Figure 4. Excitation profiles recorded at $8 \mathrm{~K}$ by monitoring at the specified emission wavelengths of the photochemical fragments of DMZ matrix isolated in argon. In panel A, the excitation spectra of the UV emission bands are shown, while panel B contains the excitation spectra of the near-UV bands.

Emission spectra were recorded with photoexcitation, at the wavelengths indicated by the arrows in Figure 2, of the new absorption features produced as a result of the previously described photolysis of the freshly deposited DMZ/Ar sample. The emission spectra resulting from excitation at wavelengths of 207 and $213 \mathrm{~nm}$ are shown in Figure 3. Strong UV and weak near-UV emission bands are observed, whose maxima are observed to shift depending on the excitation wavelength used. The emission resulting from $207 \mathrm{~nm}$ excitation comprises a pair of UV bands at 219 and $235 \mathrm{~nm}$ and a much weaker near-UV feature at $312 \mathrm{~nm}$. Excitation at $213 \mathrm{~nm}$ produces a single UV emission band at $233 \mathrm{~nm}$ and a broad, weak emission at $320 \mathrm{~nm}$ shown by the gray trace in Figure 3 .

Excitation spectra recorded for the UV and near-UV emission bands presented in Figure 3 were observed to occur in the 205220 nm spectral range, as shown in Figure 4. Panel A shows the excitation profiles of the UV 219, 233, and $235 \mathrm{~nm}$ emission bands, while panel $\mathrm{B}$ shows the excitation profiles of the weaker near-UV 312 and $320 \mathrm{~nm}$ emission bands. The excitation profile of the $233 \mathrm{~nm}$ emission extends from 202 to $218 \mathrm{~nm}$, exhibiting a dominant feature at $213 \mathrm{~nm}$, while that recorded for emission at $235 \mathrm{~nm}$ spans the same wavelength range but appears to

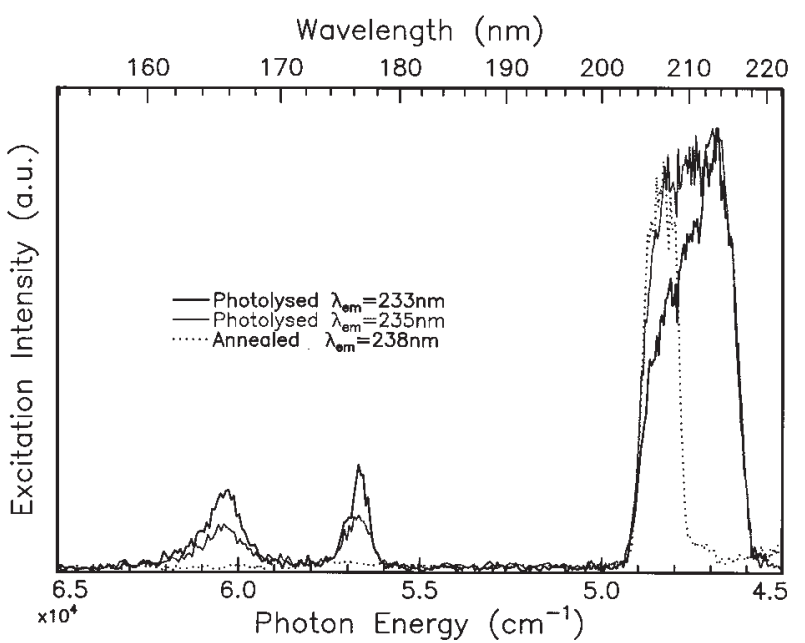

Figure 5. Higher energy excitation profiles recorded at $8 \mathrm{~K}$ for the indicated emission wavelengths of the photochemical fragments produced by zeroth-order photolysis of a 1/1000 dilution ratio DMZ/ Ar sample. The effect of annealing on this photolyzed sample to $33 \mathrm{~K}$ for a 25 min period is shown by the dotted trace. Note that the composite excitation feature centered at $213 \mathrm{~nm}$ yields the structured band at 207 $\mathrm{nm}$ after annealing. In addition, the absence of the excitation features at 165 and $176 \mathrm{~nm}$ is noteworthy, as indicated by the dotted trace.

consist of three partially resolved features at 207, 210, and 213 $\mathrm{nm}$. In contrast, a well-defined feature centered at $207 \mathrm{~nm}$ is observed when the 219 and $312 \mathrm{~nm}$ emissions are monitored as shown in panels A and B, respectively, of Figure 4. A broad excitation profile with a dominant feature located at $207 \mathrm{~nm}$ but extending up to $218 \mathrm{~nm}$ is recorded by monitoring emissions at $320 \mathrm{~nm}$.

Figure 5 shows excitation profiles recorded for the previously mentioned 233 and $235 \mathrm{~nm}$ emission bands but scanned to higher photon energy than that presented in Figure 4. Two weaker excitation features are evident in Figure 5 at 165 and $176 \mathrm{~nm}$ in addition to the broad excitation profiles in the 205$220 \mathrm{~nm}$ spectral region described above. By exciting at wavelengths of 165 or $176 \mathrm{~nm}$, emission spectra identical with that produced with $213 \mathrm{~nm}$ excitation were observed.

The observed decay time of the $235 \mathrm{~nm}$ emission resulting from $207 \mathrm{~nm}$ excitation, determined by fitting a doubleexponential function to the temporal decay profile, had a value of $1.40 \pm 0.14$ ns. The minor (3\%) component had a decay time of 0.2 ns. The temporal profile of the weaker $219 \mathrm{~nm}$ emission produced with $207 \mathrm{~nm}$ excitation resulted in a deconvoluted decay time of $0.89 \pm 0.1 \mathrm{~ns}$, which represented over $96 \%$ of the double-exponential fit. A decay time of 1.39 $\pm 0.14 \mathrm{~ns}$ was extracted from the decay curve of the $233 \mathrm{~nm}$ emission produced by excitation at $213 \mathrm{~nm}$. The temporal decay of the near-UV emission features observed in the 310-350 nm region could not be measured with the TCSPC technique, even when the storage ring was operating in the "single-bunch" mode with a repetition rate of $1.042 \mathrm{MHz}$. This behavior is indicative $\mathrm{e}^{9}$ of a long-lived emission having a lifetime greater than $10 \mu \mathrm{s}$.

C. Photolysis $(\mathbf{1 9 5} \mathbf{~ n m})$. The shapes of the 213/233 nm excitation/emission profiles were found to be strongly dependent on the type of photolysis done on DMZ. Thus, the excitation/ emission profiles recorded for DMZ/Ar following photolysis at $195 \mathrm{~nm}$ for $45 \mathrm{~min}$ are shown in Figure 6 for the indicated wavelengths. Comparing the shapes of these excitation profiles with those shown in Figure 4, for zeroth-order photolysis of a DMZ/Ar sample, it is evident that the contributions of the 207 and $210 \mathrm{~nm}$ features are much reduced in the $195 \mathrm{~nm}$ photo- 


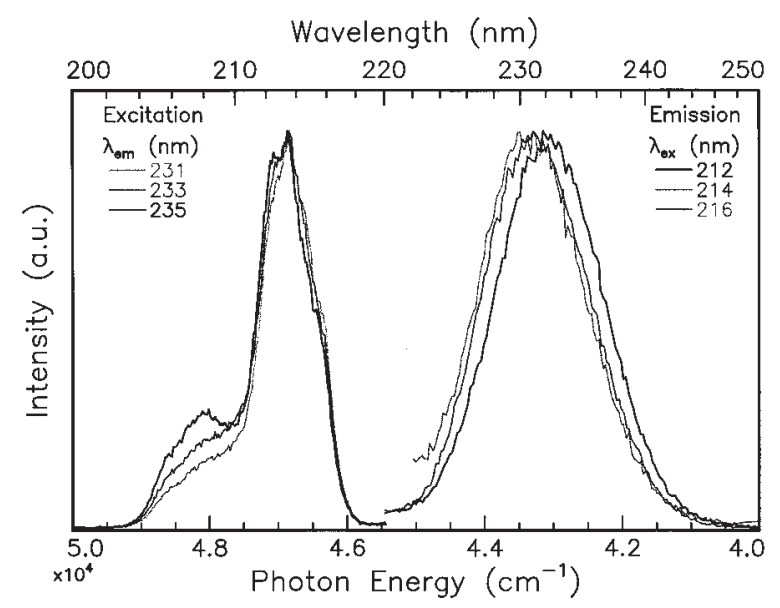

Figure 6. Excitation and emission spectra recorded at $8 \mathrm{~K}$ for a DMZ/ Ar sample after prolonged (45-min) photolysis at $195 \mathrm{~nm}$. Note the red-shift in the emission maxima as the photoexcitation energy is increased. Partially resolved features are evident at 212, 213.8, and $216 \mathrm{~nm}$ in the excitation profiles, exhibiting maxima dependent on the emission wavelength monitored.

lyzed samples, with the $213 \mathrm{~nm}$ band clearly dominating the excitation profile of the $233 \mathrm{~nm}$ emission. Hints of structure on the excitation profiles shown in Figure 6 are evident at 212, 213.8, and $216 \mathrm{~nm}$. The complexity of the emission, shown on the right of Figure 6, is evident when these particular wavelength values are selected for photoexcitation. Conspicuously, the lowest energy excitation at $216 \mathrm{~nm}$ produces the highest energy emission centered at $230 \mathrm{~nm}$, while the highest energy excitation at $212 \mathrm{~nm}$ yields the lowest energy band centered at $233 \mathrm{~nm}$. Excitation at an intermediate energy of $214 \mathrm{~nm}$ produces an emission band of intermediate energy at $231 \mathrm{~nm}$.

D. Methylzinc Radical. A careful examination was made for the production of methylzinc $\left(\mathrm{ZnCH}_{3}\right)$ upon photolysis of DMZ. This was pursued by monitoring the fluorescence ${ }^{4}$ of methylzinc in the $410-450 \mathrm{~nm}$ region by excitation of the $\tilde{A}$ ${ }^{2} \mathrm{E}_{1 / 2} \leftrightarrow \mathrm{X}^{2} \mathrm{~A}_{1} 0_{0}^{0}$ transition of $\mathrm{ZnCH}_{3}$, observed at $417.5 \mathrm{~nm}$ in the gas phase $\mathrm{e}^{7}$ and having a radiative lifetime ${ }^{2}$ of $47 \pm 2$ ns. No signal was obtained in this spectral range either with (a) wavelength-selected excitation/steady-state emission or (b) quartz-filtered zeroth-order excitation and monitoring time-gated emission in a 20-100 ns range. Thus, on the sensitivity of the present measurement, we must conclude from observation a that methylzinc is not permanently formed as a result of photodissociation of DMZ isolated in solid $\mathrm{Ar}$ and from $\mathrm{b}$ that it does not chemiluminescence if it is formed in an excited state from the dissociation of DMZ as has been reported in previous gas-phase vacuum UV measurements ${ }^{2,6}$ of dimethylcadmium using synchrotron radiation at wavelengths shorter than $205 \mathrm{~nm}$.

E. Annealing. Annealing of photolyzed DMZ/Ar samples to $33 \mathrm{~K}$ for approximately $15 \mathrm{~min}$ resulted in pronounced changes in both excitation and emission spectra. In Figure 7, the effects of annealing on the excitation spectra of the UV and near-UV emissions of the photolyzed samples are shown. In the left panel of Figure 7, the broad composite profile, shown earlier in Figure 4, recorded by monitoring emissions at 235 $\mathrm{nm}$ of the photolyzed sample is no longer present, but a single feature centered at $207 \mathrm{~nm}$ and corresponding to the excitation profile of the $219 \mathrm{~nm}$ emission results. Closer examination of the annealed $207 \mathrm{~nm}$ excitation profile shown in black on the left in Figure 7 indicates the existence of a 3-fold splitting on this band.

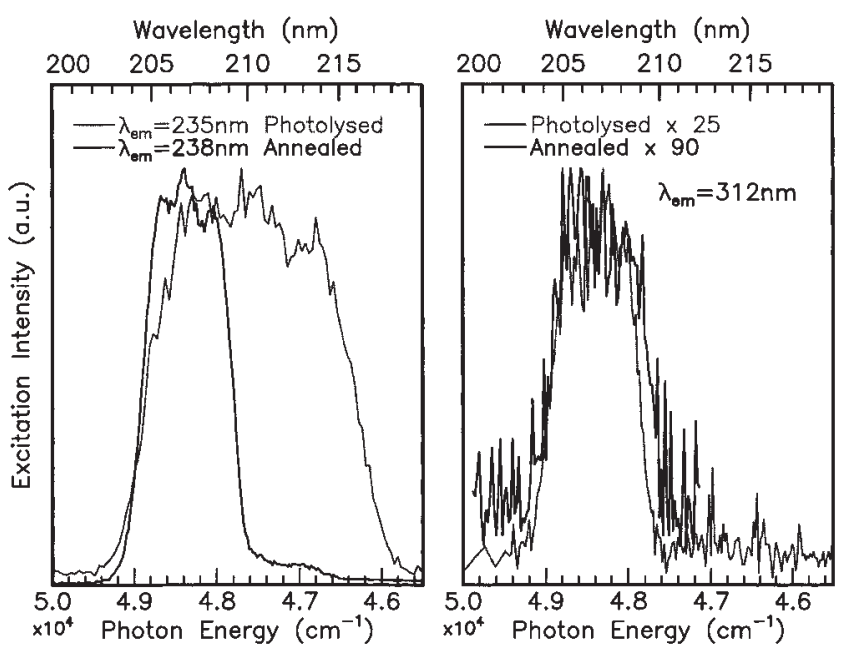

Figure 7. Excitation spectra (gray traces) recorded at $8 \mathrm{~K}$ for the UV $235 \mathrm{~nm}$ and near-UV $312 \mathrm{~nm}$ emissions, respectively, of a freshly photolyzed DMZ/Ar sample. The black traces correspond to excitation profiles recorded by monitoring the previously mentioned emissions but after sample annealing to $33 \mathrm{~K}$ for $25 \mathrm{~min}$.

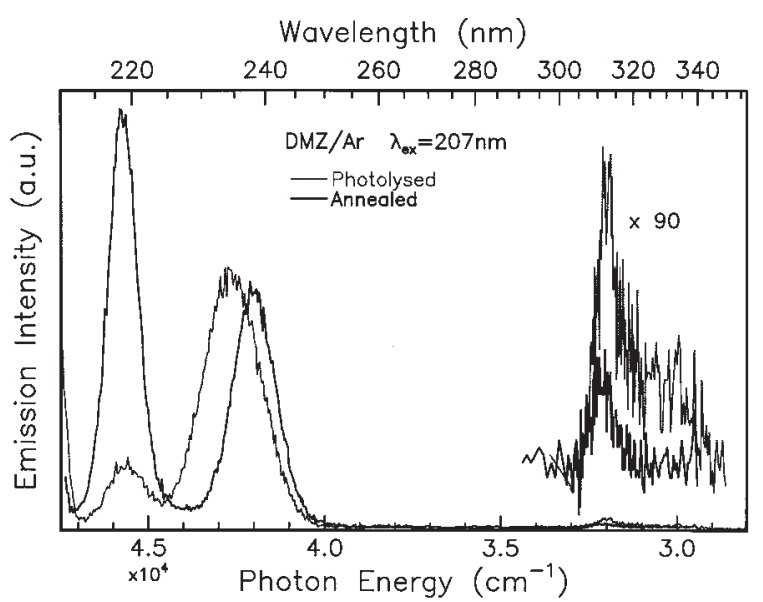

Figure 8. Emission spectra recorded at $8 \mathrm{~K}$ for a photolyzed DMZ/ Ar sample and produced using a photoexcitation wavelength of 207 $\mathrm{nm}$. The gray trace corresponds to the emission spectrum of a freshly photolyzed sample, while the black trace is the emission recorded in the same sample but annealed to $33 \mathrm{~K}$ for a period of $25 \mathrm{~min}$.

Excitation into the central component of the 3-fold splitting at $206.7 \mathrm{~nm}$ produces the emission spectrum shown by the black trace in Figure 8. A pair of UV emission bands are observed at 218.7 and $238 \mathrm{~nm}$, while a single band, weaker by a factor of 90 , is observed in the near-UV at $312 \mathrm{~nm}$. The emission bands recorded in the annealed sample (shown by the black trace in Figure 8) have a narrower bandwidth than those recorded for freshly photolyzed samples, shown by the gray trace in Figure 8. The excitation profiles of the two UV emission bands at 218.7 and $238 \mathrm{~nm}$ in the annealed sample are identical as shown by the upper traces in Figure 9. The spectral location of the excitation profile of the weaker $312 \mathrm{~nm}$ emission is, as shown on the bottom in Figure 9, the same as the UV profiles, but due to the weakness of the emission band and the resulting low signal-to-noise, the existence of splitting on the excitation band of the triplet can't be identified. However, given the similar spectral position and bandwidths of the singlet and triplet excitation bands, it is likely that the excitation profiles are the same. The excitation features present in freshly photolyzed samples at 165 and $176 \mathrm{~nm}$ were completely absent when 238 nm emission was monitored in the annealed samples, as depicted by the dotted trace in Figure 5 . 


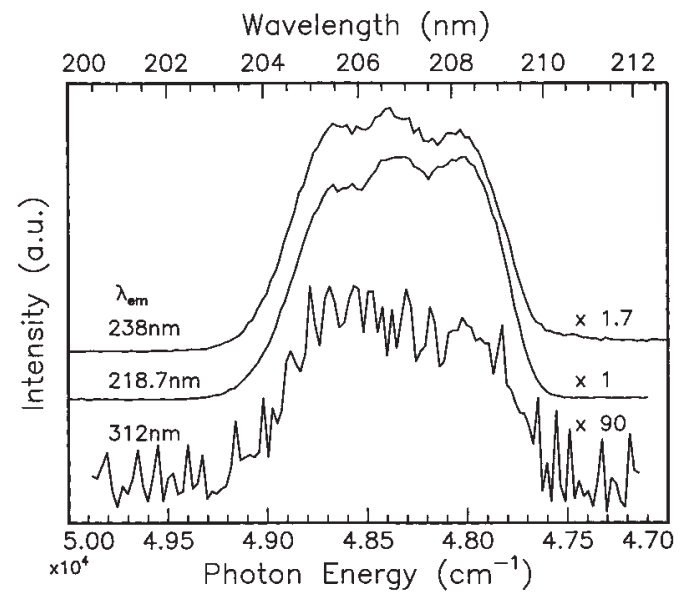

Figure 9. Comparison of the excitation spectra recorded for the three emission bands shown in Figure 8 present in DMZ/Ar samples first photolyzed and then annealed.

The decay time measured for the $218.7 \mathrm{~nm}$ emission as a result of $207 \mathrm{~nm}$ excitation was found from the TCSPC technique to be $0.92 \pm 0.1 \mathrm{~ns}$ using a double-exponential fit of the decay profile. This value was found not to vary for samples below $9 \mathrm{~K}$, prepared under the same conditions and composition, and contributed to over $97 \%$ of the decay profile. The decay profiles recorded for the $238 \mathrm{~nm}$ emission with $207 \mathrm{~nm}$ excitation exhibited an observed decay time of $1.43 \pm 0.1$ ns. Decay profiles of the $312 \mathrm{~nm}$ emission band could not be recorded with the TCSPC technique at an excitation repetition rate of $1.042 \mathrm{MHz}$, indicating ${ }^{9}$ a long-lived emission having a lifetime longer than $10 \mu \mathrm{s}$.

\section{Discussion}

A. Assignments of DMZ/Ar Absorption. Based on the similar band shapes and oscillator strengths of the DMZ absorption profiles, shown in Figure 1, recorded in solid $\mathrm{Ar}$ and in the gas phase, tentative assignments of the $\mathrm{B} \leftarrow \mathrm{X}$ and $\mathrm{A} \leftarrow \mathrm{X}$ transitions of DMZ in the matrix are made in accordance with those made by Chen and Osgood. ${ }^{5}$ It must, however, be remembered that a pronounced blue shift of $5600 \mathrm{~cm}^{-1}$ occurs in the matrix and, in contrast to the gas phase, no vibrational structure is observed on the $\mathrm{B} \leftarrow \mathrm{X}$ transition. Despite these differences, adoption of the gas-phase assignments to the matrix DMZ absorptions seems reasonable. With this assignment and on the basis of the theoretical analysis of Chen and Osgood, ${ }^{5}$ the unstructured A state of DMZ has a bent geometry, while the B state showing vibrational structure in the gas phase is linear. The large blue shifts on the two bands in the matrix can be traced back to the dominant Rydberg characteristics of the excited A and B states of DMZ. It is known from $a b$ initio calculations $^{2}$ that the excited electronic states of DMZ have this characteristic and from previous matrix work ${ }^{10}$ on NO that Rydberg states exhibit such large blue shifts in the solid rare gases. This strong interaction between the DMZ guest and its host also explains the lack of vibrational structure on the B $\longleftarrow$ $\mathrm{X}$ transition since the accompanying strong electron-phonon coupling will broaden the vibrational bands into a smooth profile.

B. Atomic Zinc Isolated in Annealed Solid Argon. A summary of the excitation and emission spectra recorded in the UV region for an annealed, previously-photolyzed DMZ/Ar sample is presented on the bottom in Figure 10. The upper excitation/emission profiles in Figure 10 were recorded for a sample prepared by the co-deposition of atomic zinc vapor with argon in a dilution ratio of $1 / 10^{4}$ and reported by us in a previous publication. ${ }^{9}$ Shown for comparison is the position of the $4 \mathrm{~s} 4 \mathrm{p}$

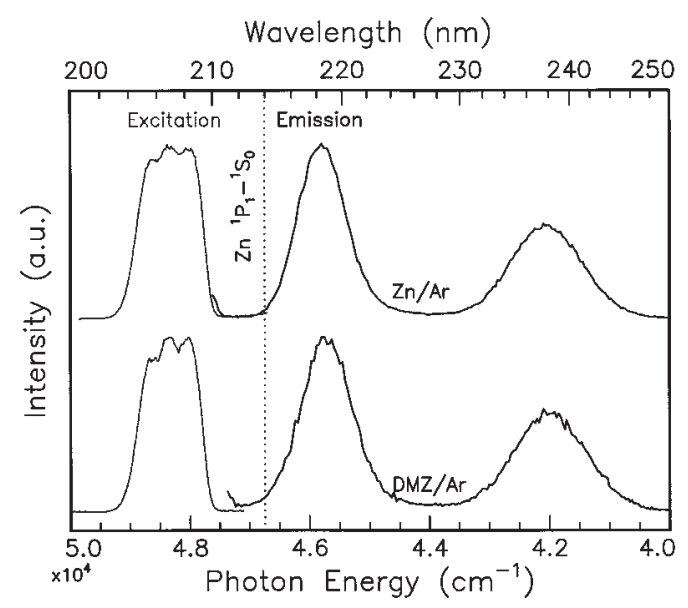

Figure 10. Excitation spectra recorded for the $218.7 \mathrm{~nm}$ emission in $\mathrm{Zn} / \mathrm{Ar}$ and photolyzed DMZ/Ar samples are shown in gray on the extreme left-hand side. The emission spectra recorded at $8 \mathrm{~K}$ due to $207 \mathrm{~nm}$ excitation of the aforementioned samples are shown on the right-hand side in black. The position of the singlet resonance transition of atomic zinc in the gas phase is indicated by the vertical dotted lines for comparison.

${ }^{1} \mathrm{P}_{1}-4 \mathrm{~s}^{2}{ }^{1} \mathrm{~S}_{0}$ singlet resonance transition of atomic zinc in the gas phase. The excitation feature centered at $207 \mathrm{~nm}$ in $\mathrm{Zn} / \mathrm{Ar}$ and photolyzed DMZ/Ar samples which exhibits a 3-fold splitting corresponds to the absorption spectra recorded by several groups ${ }^{11}$ for zinc in argon and assigned to the $4 \mathrm{~s} 4 \mathrm{p}^{1} \mathrm{P}_{1}$ $\leftarrow 4 \mathrm{~s}^{2}{ }^{1} \mathrm{~S}_{0}$ transition of $\mathrm{Zn}$.

Observed decay times of $0.91 \pm 0.1$ and $1.43 \pm 0.14$ ns were recorded for the 218.7 and $238 \mathrm{~nm}$ emissions respectively due to excitation at $207 \mathrm{~nm}$ and found independent of temperature below $9 \mathrm{~K}$. These values are identical within experimental error to those recorded by $\mathrm{us}^{9}$ for the UV emission bands in pure $\mathrm{Zn} / \mathrm{Ar}$ samples and assigned to the singlet emission of atomic zinc in Ar. The presence of the pair of singlet emission bands has been attributed ${ }^{12}$ to the co-existence of energetically distinct minima on the potential energy surface arising from Jahn-Teller coupling of the Ar lattice modes and the $4 \mathrm{~s} 4 \mathrm{p}{ }^{1} \mathrm{P}_{1}$ state of atomic zinc. Excellent agreement clearly exists between the steadystate and time-resolved measurements in pure $\mathrm{Zn} / \mathrm{Ar}$ samples and the annealed, photolyzed DMZ/Ar samples, confirming the formation of atomic zinc as a dissociation product of the photolysis of DMZ in solid argon.

C. Triplet Emission. Although the singlet UV emissions of atomic zinc in $\mathrm{Zn} / \mathrm{Ar}$ and photolyzed DMZ/Ar samples are very similar, the intensity of the emission at $312 \mathrm{~nm}$ was observed to be considerably enhanced in the photolyzed DMZ/ Ar samples, because, as shown by trace A on the top in Figure 11 , this band was not detected in the pure $\mathrm{Zn} / \mathrm{Ar}$ samples. The spectral location and long ( $>10 \mu$ s) decay time of the $312 \mathrm{~nm}$ emission strongly suggests that this band corresponds to the $4 \mathrm{p}$ ${ }^{3} \mathrm{P}_{1} \rightarrow 4 \mathrm{~s}{ }^{1} \mathrm{~S}_{0}$ phosphorescence transition of atomic zinc occurring at $307.6 \mathrm{~nm}$ in the gas phase ${ }^{13}$ and having a radiative lifetime ${ }^{14}$ of $30.4 \mu \mathrm{s}$. Stronger $\mathrm{Zn}\left({ }^{3} \mathrm{P}_{1}\right) / \mathrm{Ar}$ emission was observed, as shown in trace C of Figure 11, in photolyzed DMZ/ Ar samples in which the DMZ was not subjected to purification with freeze-pump-thaw (FPT) cycles. Such unpurified samples are known from IR absorption measurements ${ }^{8}$ to be contaminated with methane, and as a check of the role played by the hydrocarbon in the triplet enhancement, samples formed by the co-deposition of atomic zinc vapor with $\mathrm{Ar}$ doped with $\mathrm{CH}_{4}$ and $\mathrm{C}_{2} \mathrm{H}_{6}$ were analyzed. The results are shown in the lower two panels of Figure 11.

It is evident in trace B of Figure 11 that the $312 \mathrm{~nm}$ band is present, albeit weak compared to the $5 \%$ alkane/Ar samples, in 


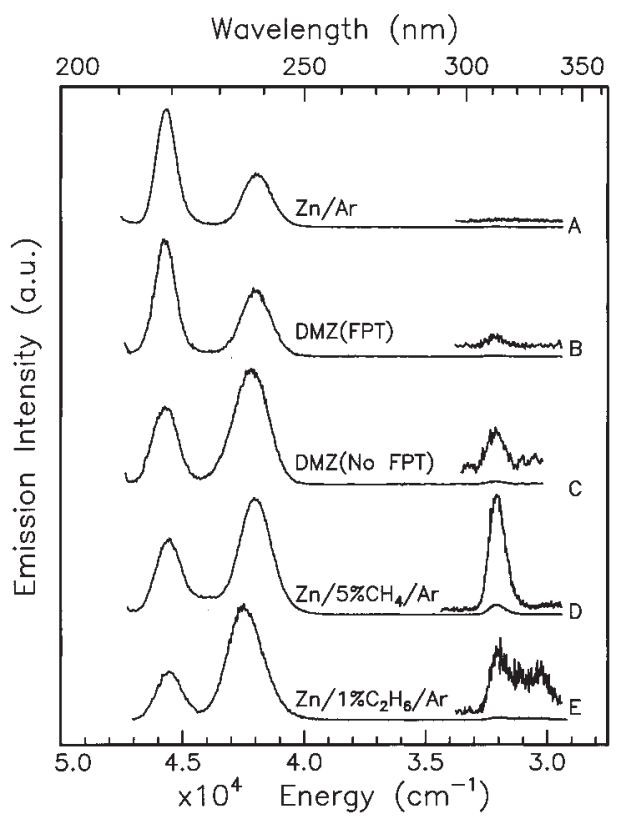

Figure 11. Emission spectra recorded as a result of atomic $\mathrm{Zn}$ excitation at $206.7 \mathrm{~nm}$ in pure Ar samples and matrices doped with the specified hydrocarbons indicating the increased intensity of the 312 $\mathrm{nm}$ band. Note the absence of the emission band in the pure $\mathrm{Zn} / \mathrm{Ar}$ samples and its presence in photolyzed DMZ/Ar and hydrocarbon-doped Ar samples. The pair of triplet emission bands evident in trace E arise from different site occupancies of atomic zinc.

photolyzed DMZ/Ar samples prepared from purified DMZ. IR measurements show that no methane persists the FPT cycles, yet the enhancement of the triplet emission still exists in these DMZ/Ar samples. The reason for the enhancement is evident in the IR spectra ${ }^{8}$ of the annealed, previously photolyzed DMZ/ Ar samples which show the growth of ethane bands upon annealing. In the bottom panel of Figure 11, the emission spectrum of a $1 \%$ ethane/Ar sample is shown, indicating the presence of the weak $312 \mathrm{~nm}$ band. The more intense triplet atomic zinc emission in photolyzed DMZ/Ar samples relative to pure $\mathrm{Zn} / \mathrm{Ar}$ samples is explained in terms of the enhanced intersystem crossing occurring in the presence of hydrocarbon species $^{15}$ in the former samples.

D. “Perturbed” Atomic Zinc. By exciting at 165, 176, and $213 \mathrm{~nm}$ in freshly photolyzed DMZ/Ar samples, identical emission spectra were produced with bands at 233 and $320 \mathrm{~nm}$. Excitation at $207 \mathrm{~nm}$ produced emission bands at different positions, namely, a pair of UV emissions at 219 and $235 \mathrm{~nm}$ and near-UV emission at $312 \mathrm{~nm}$ as shown previously by the black trace in Figure 3. This behavior would suggest that one species is responsible for the 233-nm emission and a different species is responsible for the emissions at 219 and $235 \mathrm{~nm}$. Examination of the excitation profiles of the UV and near-UV emissions shown in Figure 4 reinforces this suggestion. In particular, the similar excitation profiles centered at $207 \mathrm{~nm}$ recorded for emission monitored at 219 and $312 \mathrm{~nm}$ can, from a comparison of annealed DMZ/Ar samples and pure $\mathrm{Zn} / \mathrm{Ar}$ samples, ${ }^{9}$ be attributed to atomic zinc fully isolated in the $\mathrm{Ar}$ lattice. In contrast to the $207 \mathrm{~nm}$ band, broad, ill-defined excitation profiles, extending over a larger wavelength range of $205-220 \mathrm{~nm}$, were recorded when monitoring the 233 and $320 \mathrm{~nm}$ emissions also shown in Figure 4.

The decay times recorded for the $233 \mathrm{~nm}$ emission produced with excitation at 213,176 , or $165 \mathrm{~nm}$ were found to be identical, within the experimental error of the present lifetime measurements. Thus, these excitation features are assigned to transitions of a species distinct from that associated with the 207-nm excitation feature identified above as being atomic zinc fully isolated in Ar.

So far, the emission bands at 233 and $320 \mathrm{~nm}$ and their corresponding excitation spectra observed in freshly photolyzed samples have been attributed to a single species but are not assigned. The obvious candidates for assignment of this species are, in the order of their importance, clusters, site effects, or different photochemical products. Note that the species described above results from photolysis of DMZ monomer isolated in solid $\operatorname{argon}^{16}$ so that the cluster assignment can be ruled out immediately. Moreover, absorption spectra of zinc aggregates in solid neon ${ }^{17}$ and xenon ${ }^{11}$ have been characterized and previously published. It is found that the spectral positions of low-order aggregates do not coincide with any of the bands observed in this study. The broad excitation features shown by panel A in Figure 4 between 205 and $220 \mathrm{~nm}$ and the features at 165 and $176 \mathrm{~nm}$ shown in Figure 5 disappear upon sample annealing, leaving only the 3 -fold split excitation feature centered at $207 \mathrm{~nm}$ as shown in Figure 10. In a parallel FTIR study of DMZ/Ar photolyzed at $193 \mathrm{~nm}$ using an ArF excimer laser, ${ }^{8}$ methyl radical strongly perturbed by a neighboring species was identified at $730.4 \mathrm{~cm}^{-1}$. Upon annealing, the perturbed methyl radical was observed to disappear with the concomitant production of the stable molecule ${ }^{18}$ ethane.

The luminescence spectroscopy of photolyzed DMZ/Ar samples recorded in the present study indicates the production of atomic $\mathrm{Zn}$ isolated in solid Ar upon sample annealing. Taking this in conjunction with the pronounced annealing effects observed in the IR study, the natural assignment of the 233/ 320-nm emission bands observed in the present UV luminescence measurements is a zinc atom interacting strongly with a nearby methyl radical but not forming a $\mathrm{Zn}-\mathrm{C}$ bond to give methylzinc. The excitation features at 165, 178, and $213 \mathrm{~nm}$ of photolyzed DMZ/Ar samples, all of which have emission decay times similar to atomic zinc, disappear upon annealing, as evidenced in Figure 5, and are thereby ascribed to "perturbed" atomic zinc. The existence of a zinc atom in the presence of a very reactive methyl radical, which might initially seem surprising, will now be discussed. This species shall be referred to as "perturbed" atomic zinc throughout the remainder of this paper.

The co-existence of atomic zinc in the vicinity of two methyl radicals is realized because the zinc atom is formed in the unreactive ${ }^{1} \mathrm{~S}_{0}$ ground state. Based on valence bond theory, formation of a chemical bond between atomic zinc and the methyl radical requires hybridization of the $4 \mathrm{~s}$ and $4 \mathrm{p}$ orbitals of atomic zinc. High-level $a b$ initio calculations ${ }^{19,20}$ on $\mathrm{ZnCH}_{3}$ verify this simple valence bond model, indicating predominant $4 \mathrm{~s}(\mathrm{Zn}) 4 \mathrm{p}_{\mathrm{z}}(\mathrm{Zn})$ character in the highest occupied molecular orbital. Hybridization of the $4 \mathrm{~s} / 4 \mathrm{p}$ orbitals of atomic zinc in the low-temperature matrix is not feasible because the promotion energy required is far too great to be provided by the available thermal energy. Hence, the closed subshell electronic configuration of atomic zinc in the ${ }^{1} \mathrm{~S}_{0}$ ground state does not react chemically with the methyl radical to form the $\mathrm{Zn}-\mathrm{C}$ bond in methylzinc, but its spectroscopy is strongly perturbed by a nearby methyl radical. This situation can be ascribed to the formation of the van der Waals complex $\mathrm{Zn} \cdot \mathrm{CH}_{3}$. An important mechanistic consequence of the presence of the "inert" zinc atom positioned between the methyl radicals is that it impedes the latters' approach in the cylindrical divacancy site in the Ar lattice and thereby prohibits their recombination to form vibrationally excited ethane. This point will be elaborated upon in the section dealing with the photodissociation mechanism of DMZ in solid argon. 
The complex nature of the 213/233 nm excitation/emission profiles, depicted in Figure 6, indicates that the perturbed species does not exist in a single form. In particular, the red-shifting of the emission maxima with increasing photoexcitation energy and the hint of structure on the excitation profiles indicates that homogeneous linewidths have not been recorded. It would appear from the excitation profiles that at least three forms of the perturbed $\mathrm{Zn} \cdot \mathrm{CH}_{3}$ complex are produced with the photodissociation of matrix-isolated DMZ. The origin of the triplication cannot be identified from the present IR or UV luminescence measurements, but the likely candidates would be different site occupancies or distinct separation or orientations of the methyl group and atomic zinc in the weakly bound $\mathrm{Zn} \cdot \mathrm{CH}_{3}$ complex.

In support of the perturbed $\mathrm{Zn}$ assignment, the absence in the present study of the methyl radical absorption at $150 \mathrm{~nm}$, previously observed in solid argon by Milligan and Jacox, ${ }^{21}$ indicates that isolated methyl radicals are not produced from the dissociation of matrix-isolated DMZ. Furthermore, the predissociative methyl transition observed by Herzberg ${ }^{22}$ near $215 \mathrm{~nm}$ in the gas phase was not observed in the Ar matrix by Milligan and Jacox. ${ }^{21}$ Accordingly, the excitation features observed between 205 and $220 \mathrm{~nm}$ in a freshly photolyzed sample are not assigned to isolated methyl radical but to the ${ }^{1} \mathrm{P}_{1} \leftarrow{ }^{1} \mathrm{~S}_{0}$ transition of atomic zinc perturbed strongly with a methyl radical. This assignment is consistent with the $1.40 \mathrm{~ns}$ lifetime observed for the $233 \mathrm{~nm}$ emission, a value identical to that recorded ${ }^{9}$ in pure $\mathrm{Zn} / \mathrm{Ar}$ samples and which agrees well with the gas-phase radiative lifetime ${ }^{14}$ of $1.41 \mathrm{~ns}$ for the ${ }^{1} \mathrm{P}_{1} \rightarrow$ ${ }^{1} \mathrm{~S}_{0}$ transition of atomic zinc.

Examination of the higher energy levels of atomic zinc show that many states exist in the same region as the excitation features at 165 and $176 \mathrm{~nm}$, none of which can be accessed by electric-dipole transitions from the ${ }^{1} \mathrm{~S}_{0}$ ground state in the gas phase. Possible assignments for the 176 and $165 \mathrm{~nm}$ excitation bands may be the spin-forbidden ${ }^{3} \mathrm{~S}_{1} \leftarrow{ }^{1} \mathrm{~S}_{0}$ transition, occurring at $186.3 \mathrm{~nm}$, and the parity-forbidden ${ }^{1} \mathrm{D}_{2} \leftarrow{ }^{1} \mathrm{~S}_{0}$ transition at $163 \mathrm{~nm}$. These transitions, strictly forbidden for the free zinc atom in the gas phase, may become partially allowed due to a lowering of symmetry in the perturbed $\mathrm{Zn} \cdot \mathrm{CH}_{3}$ complex generated upon dissociation of DMZ. In the truly isolated form of atomic $\mathrm{Zn}$ in solid Ar, cubooctahedral symmetry will exist for the $\mathrm{Zn}$ atom isolated in a single substitutional site in the $\mathrm{Ar}$ lattice, a situation more akin to the full rotational symmetry of the gas phase. Thus, it is expected that the transitions forbidden in the gas phase will also not be observed in annealed $\mathrm{Ar}$ samples.

E. Dissociation Mechanism. The quartz optical filter used in the zero-order photolysis selects wavelengths longer than 155 $\mathrm{nm}$ of the undispersed synchrotron radiation. Thus, energies of up to $184 \mathrm{kcal} / \mathrm{mol}$ are available to induce photodissociation of DMZ in the solid, but since only $63.7 \mathrm{kcal} / \mathrm{mol}$ is required to break the first zinc-methyl bond, ${ }^{23}$ an excess of approximately $120 \mathrm{kcal} / \mathrm{mol}$ is available to break the second zincmethyl bond of $25 \mathrm{kcal} / \mathrm{mol}$ ! The energy made available by the type of UV photolysis applied is therefore much more than what is required to break both methyl-zinc bonds in either a sequential or a concerted fashion. Due to solid-state cage effects, however, direct cage exit of the cleaved methyl radical produced in the stepwise mechanism will be hindered, forcing it and methylzinc radical to occupy the same cage. Recombination of the methyl and methylzinc radicals will occur to regenerate DMZ. A concerted mechanism will, in contrast, be expected to achieve permanent dissociation, as the two methyl

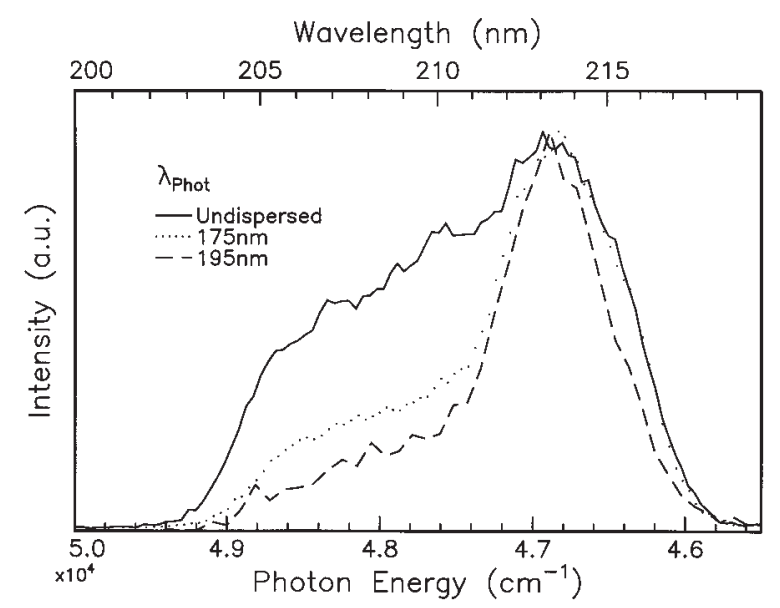

Figure 12. Excitation profiles recorded for the $233 \mathrm{~nm}$ emission of DMZ/Ar samples after 10 min photolysis at the specified wavelengths. Note the increasing intensity of the $207 \mathrm{~nm}$ feature as the photolysis energy is increased.

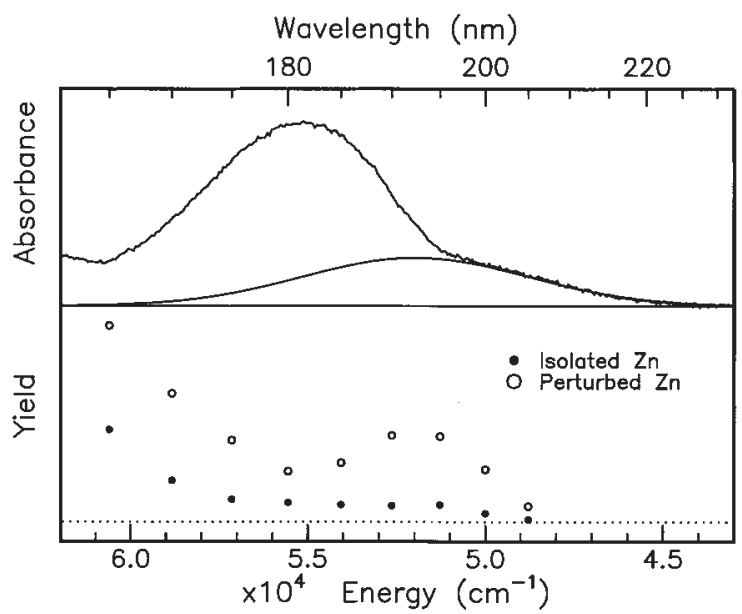

Figure 13. Growth curves obtained for the production of isolated atomic zinc (ex/em $=207 / 219 \mathrm{~nm}$ ) and perturbed atomic zinc (ex/em $=213 / 233 \mathrm{~nm}$ ) as a function of the photolysis energy. Shown in the upper panel is the absorption spectrum and spectral assignments of the $\mathrm{B} \leftarrow \mathrm{X}$ and $\mathrm{A} \leftarrow \mathrm{X}$ transitions of DMZ/Ar. Note the inefficiency of the direct production of isolated atomic until a photolysis wavelength of $180 \mathrm{~nm}$ is reached.

radicals will then be separated by an unreactive ground-state zinc atom.

The recorded excitation spectra indicate that only a small amount of atomic zinc exits the cage directly, facilitated by the $95 \mathrm{kcal} / \mathrm{mol}$ of excess internal energy resulting from the use of the quartz filter to select the photolysis wavelengths for the photodissociation of DMZ. When the kinetic energy of the fragments is lowered, by photolysis at $175 \mathrm{~nm}$, the amount of isolated atomic zinc, centered at $207 \mathrm{~nm}$, decreases dramatically, as shown in Figure 12. For photolysis wavelengths longer than $175 \mathrm{~nm}$ at 192 and $200 \mathrm{~nm}$, the production of truly isolated atomic zinc is much reduced. Therefore, it would appear that little direct cage exit of atomic $\mathrm{Zn}$ occurs at these lower energies.

Growth curves indicating the yields of isolated atomic zinc and perturbed atomic zinc as a function of photolysis wavelength are presented in Figure 13 along with the absorption profile of DMZ/Ar. The growth curve of the perturbed species is prompt, showing a maximum at around $193 \mathrm{~nm}$ and showing a second onset for wavelengths shorter than $180 \mathrm{~nm}$. In contrast, the yield of isolated atomic zinc is negligible until around $175 \mathrm{~nm}$ (163.4 kcal/mol), thereafter increasing steadily to the quartz cut- 
off. From a comparison of these growth curves with the absorption profile, shown in the upper panel of Figure 13, it appears that the production of the perturbed atomic zinc species follows the A-state absorption profile. In contrast, the production of isolated atomic zinc has a threshold at $175 \mathrm{~nm}$ on the blue wing of the B-state absorption.

On the basis of the spectral assignments made in section A of the Discussion section, one would expect, as observed experimentally for dimethylcadmium, ${ }^{6}$ that the bent A state would be more likely to produce translationally hot atomic zinc than the B state, with its linear geometry. From a comparison of the fragment growth curves and the absorption curves shown in Figure 13, the opposite behavior is exhibited in the matrix. This difference is probably a reflection of the influence the surrounding host matrix has on the production of the isolated fragment atomic zinc, requiring a very large excess of dissociation energy for the direct production of such a species.

Assuming that all the excess internal energy goes into the kinetic energy of the escaping $\mathrm{Zn}$ atom, then the energy threshold for direct cage escape of atomic $\mathrm{Zn}$ from the growth curve in Figure 12 is between 75 and $60 \mathrm{kcal} / \mathrm{mol}$. If, more realistically, it is assumed that equal partitioning of the excess internal energy occurs between the three fragments-the two methyl radicals and the zinc atom-then a threshold range of between 25 and $20 \mathrm{kcal} / \mathrm{mol}$ is obtained. Having no energetically accessible internal degrees of freedom, all of this energy will go into the kinetic energy of the $\mathrm{Zn}$ atom, while in the methyl groups it must be shared with vibrational excitation. This may explain why the methyl groups do not escape from the original site of isolation while the $\mathrm{Zn}$ atom does.

During sample annealing, the increased thermal energy and expansion of the host's lattice facilitates the mobility of photochemical fragments and the possible recombination of the two methyl radicals. Expansion of the host's lattice allows enough space within the divacancy site originally occupied by the parent DMZ molecule for the two methyl radicals initially separated by the inert zinc atom to approach and combine. From gas phase ${ }^{24}$ and theoretical work, ${ }^{25}$ it is known that direct recombination of methyl radicals occurs without any activation energy barrier to produce ethane, with $87.6 \mathrm{kcal} / \mathrm{mol}$ of vibrational excitation. ${ }^{26}$ The FTIR study of DMZ/Ar which follows indicates that this excess energy is dispersed through two channels, namely, stabilization of ethane by energy dissipation to the lattice or further dissociation to form the new products $^{27}$ ethylene and molecular hydrogen. Supporting the IR observations, molecular hydrogen gas has been monitored by mass spectrometry escaping from the Ar lattice during annealing in this study.

\section{Conclusions}

Using steady-state and time-resolved optical luminescence spectroscopy, the photochemical fragments atomic zinc perturbed by a nearby methyl radical and truly isolated atomic zinc have been identified from the photodissociation of DMZ in an argon matrix. Excitation spectra of truly isolated atomic zinc in an argon matrix show a 3-fold splitting attributed to the dynamical Jahn-Teller effect. These splittings are identified as the matrix equivalent of the gas-phase $4 \mathrm{p}^{1} \mathrm{P}_{1} \leftarrow 4 \mathrm{~s}{ }^{1} \mathrm{~S}_{0}$ resonance transition of atomic zinc. From a combination of luminescence spectroscopy and time-resolved measurements, singlet and triplet transitions were assigned respectively to the pair of UV emission bands at 218.7 and $238 \mathrm{~nm}$ and a nearUV emission band at $312 \mathrm{~nm}$ of matrix-isolated zinc in argon.

A concerted loss mechanism of the methyl groups is ascribed as being responsible for the permanent photodissociation of
DMZ in solid Ar. It is proposed that both methyl radicals occupy the extreme positions in the divacancy site originally occupied by the parent DMZ molecule, with the perturbed zinc atom positioned between. Atomic zinc is unreactive with respect to the methyl radicals because it is formed in the ${ }^{1} S_{0}$ ground state, a closed subshell electronic configuration allowing it to co-exist in the vicinity of a methyl radical. This proposal is confirmed by a complementary FTIR study of DMZ/Ar, ${ }^{8}$ where the species methyl radical strongly interacting with a nearby zinc atom has been characterized. The photochemical fragment methylzinc radical may be produced, but due to solidstate cage effects, the first cleaved methyl radical is hindered from exiting the cage. Thus, geminate recombination of the methyl and methylzinc radicals occurs, regenerating the DMZ molecule in the matrix cage.

In the case of high-energy photolysis $(\lambda<175 \mathrm{~nm})$ of DMZ/ Ar, direct cage exit of the zinc atom is facilitated with an equal three-way partitioning of the excess energy between the two methyl radicals and a zinc atom. The two remaining photofragment methyl radicals occupy the original site of isolation of the DMZ molecule and subsequently combine along a barrierless surface to form ethane. Small amounts of ethane were produced as indicated by triplet emission enhancement for samples of atomic zinc isolated with different concentrations of ethane dopant in argon matrices. An enhancement in the relaxation of the singlet excited state of atomic zinc to the triplet occurs in the presence of the ethane. This ISC competes very efficiently with chemical quenching of singlet excited zinc which, as presented in the following paper in this issue, results in the insertion of a zinc atom into a $\mathrm{C}-\mathrm{H}$ bond of ethane to form ethylzinc hydride. Although a definite mechanism for the ISC enhancement in the presence of the hydrocarbons has not been established, it is proposed, as observed in earlier $\mathrm{Mg} / \mathrm{CH}_{4}$ work, ${ }^{28}$ that the enhanced production of triplet emission is a result of the initially attractive inserted reaction coordinate.

Acknowledgment. We acknowledge W. Laasch, S. Moeller, P. Kerins, and B. Healy for their technical assistance in this work. This research was funded by the European Union HC\&M 1993-’95 “Access to Large Scale Facilities” Program.

\section{References and Notes}

(1) Ehrlich, D. J.; Osgood, R. M. Chem. Phys. Lett. 1981, 79, 38.

(2) Ibuki, T.; Hiraya, A.; Shobatake, K. J. Chem. Phys. 1990, 92, 2797.

(3) Young, P. J.; Gosavi, R. K.; Connor, J.; Strausz, O. P.; Gunning, H. E. J. Chem. Phys. 1973, 58, 5280.

(4) Jackson, R. L. Chem. Phys. Lett. 1990, 174, 53.

(5) Chen, C. J.; Osgood, R. M. J. Chem. Phys. 1984, 81, 327.

(6) Yu, C. F.; Young, F.; Tsukiyama, K.; Bersohn, R.; Preses, J. J. Chem. Phys. 1986, 85, 1382. Penner, A.; Amirav, A.; Bersohn, R. Chem. Phys. Lett. 1991, 176, 147.

(7) Robles, E. S. J.; Ellis, A. M.; Miller, T. A. Chem. Phys. Lett. 1991, 178, 185. Cerny, T. M.; Tan, X. Q.; Williamson, J. M.; Robles, E. S. J.; Ellis, A. M.; Miller, T. A. J. Chem. Phys. 1993, 99, 9376.

(8) Bracken, V. A.; Legay-Sommaire, N.; McCaffrey, J. G. J. Phys. Chem. A 1997, 101, 9863.

(9) Bracken, V. A.; Gürtler, P.; McCaffrey, J. G. J. Chem. Phys. 1997, 107, 5290; 1997, 107, 5300.

(10) Chergui, M.; Schwentner, N. J. Chem. Phys. 1992, 97, 2881.

(11) Hoffmann-Millack, B.; Klein, A.; Lagier, H.; Maid, B.; Hormes, J. Chem. Phys. 1989, 136, 453 and references therein.

(12) McCaffrey, J. G.; Kerins, P. J. Chem. Phys. 1997, 106, 9745.

(13) Moore, C. E. Atomic Energy Levels; US GPO: Washington, DC, 1952.

(14) Fuhr, J. R.; Wiese, W. L. Atomic Transition Probabilities, 10-179. In CRC Handbook of Chemistry and Physics, 73rd ed.; Lide, D. R., Ed.; CRC: Boca Raton, FL, 1991-1993.

(15) Bracken, V. A. Ph.D. Thesis, National University of Ireland, Maynooth, 1997.

(16) The isolation of DMZ in monomer and cluster form has been established in our FTIR spectroscopic study presented in paper 2 (ref 8). 
(17) Schroeder, W.; Wiggenhauser, H.; Schrittenlacher, W.; Kolb, D. M. J. Chem. Phys. 1987, 86, 1147.

(18) Upon annealing, new bands were observed at 820.4, 1374.5, and $1466.1 \mathrm{~cm}^{-1}$ which coincide exactly with the frequencies of the $v_{9}, v_{6}$, and $v_{8}$ modes of ethane/Ar, respectively; 948.1 and $1438.9 \mathrm{~cm}^{-1}$ the $v_{7}$ and $v_{12}$ modes of ethylene/Ar; and $736.8 \mathrm{~cm}^{-1}$ the $\nu_{5}$ mode of acetylene.

(19) Kaupp, M.; Stoll, H.; Preuss, H. J. Comput. Chem. 1990, 11, 1029.

(20) Ab initio calculation of the reaction coordinate for the insertion of a $\mathrm{Zn}$ atom into a $\mathrm{C}-\mathrm{H}$ bond of methane by Novaro and co-workers shows an activation energy barrier of $88 \mathrm{kcal} / \mathrm{mol}$ on the ground-state surface correlating with the $(4 \mathrm{~s})^{2}{ }^{1} \mathrm{~S}_{0}$ state of atomic Zn. Castillo, S.; RamirezSolis, A.; Dias, D.; Poulain, E.; Novaro, O. Mol. Phys. 1994, 81, 825.

(21) Milligan, D. E.; Jacox, M. E. J. Phys. Chem. 1967, 47, 5146.
(22) Herzberg, G.; Shoosmith, J. Can. J. Phys. 1956, 34, 523. Herzberg, G. Proc. R. Soc. London 1961, A262, 291.

(23) Jackson, R. L. Chem. Phys. Lett. 1990, 174, 53.

(24) Slagle, I. R.; Gutman, D.; Davies, J. W.; Pilling, M. J. J. Phys. Chem. 1988, 92, 2455.

(25) Wagner, A. F.; Wardlaw, D. M. J. Phys. Chem. 1988, 92, 2462. Wardlaw, D. M.; Marcus, R. A. J. Chem. Phys. 1985, 83, 3462.

(26) Baulch, D. L.; Cox, R. A.; Hampson, R. F.; Kerr, J. A.; Troe, J.; Watson, R. T. J. Phys. Chem. Ref. Data 1984, 13, 1259.

(27) The small amounts of acetylene observed in the FTIR study arise from ArF photolysis of ethylene.

(28) McCaffrey, J. G.; Ozin, G. A. J. Chem. Phys. 1989, 89, 1844. 